\title{
Single cavitation bubble generation and observation of the bubble collapse flow induced by a pressure wave
}

\author{
Sheng-Hsueh Yang $\cdot$ Shenq-Yuh Jaw $\cdot$ \\ Keh-Chia Yeh
}

Received: 3 November 2008/Revised: 25 April 2009/Accepted: 27 April 2009/Published online: 15 May 2009

(C) The Author(s) 2009. This article is published with open access at Springerlink.com

\begin{abstract}
This study utilizes a U-shape platform device to generate a single cavitation bubble for a detailed analysis of the flow field characteristics and the cause of the counter jet during the process of bubble collapse caused by sending a pressure wave. A high speed camera is used to record the flow field of the bubble collapse at different distances from a solid boundary. It is found that a Kelvin-Helmholtz vortex is formed when a liquid jet penetrates the bubble surface after the bubble is compressed and deformed. If the bubble center to the solid boundary is within one to three times the bubble's radius, a stagnation ring will form on the boundary when impinged by the liquid jet. The fluid inside the stagnation ring will be squeezed toward the center of the ring to form a counter jet after the bubble collapses. At the critical position, where the bubble center from the solid boundary is about three times the bubble's radius, the bubble collapse flow will vary. Depending on the strengths of the pressure waves applied, the collapse can produce a Kelvin-Helmholtz vortex, the Richtmyer-Meshkov instability, or the generation of a counter jet flow. If the bubble surface is in contact with the solid boundary, the liquid jet can only move inside-out without producing the stagnation
\end{abstract}

\footnotetext{
S.-H. Yang $(\bowtie) \cdot$ K.-C. Yeh

Department of Civil Engineering,

National Chiao Tung University,

30010 Hsinchu, Taiwan, ROC

e-mail: shyang.cv91g@nctu.edu.tw

K.-C. Yeh

e-mail: kcyeh@mail.nctu.edu.tw

S.-Y. Jaw

Department of Systems Engineering and Naval Architecture,

National Taiwan Ocean University, 20224 Keelung,

Taiwan, ROC

e-mail: syjaw@ntou.edu.tw
}

ring and the counter jet; thus, the bubble collapses along the radial direction. The complex phenomenon of cavitation bubble collapse flows is clearly manifested in this study.

\section{Introduction}

Extensive studies in the past have recorded the fact that cavitation bubbles could be the cause of damage to the surfaces of pressure conduits and turbine blades of hydraulic machinery. Some observations have revealed that when cavitation bubbles near the solid surfaces break up, they may form strong so-called water-harming type of pressure waves. The repeated impingements of these strong shock waves can result in varying degree of damages to the walls of the surrounding structures.

The possibility of serious structural damage caused by these tiny cavitation bubbles has surely caught the attention and curiosity of researchers. Many of them have plunged into the study of the characteristics of the flow field of bubble collapse and its effect on the deterioration and destruction of its surrounding solid boundary. These studies include the understanding of the shock wave, the characteristics of the resultant luminescence, and the jetrelated fields. If the cavitation bubble is located near a solid boundary at a certain suitable distance, it is possible that the production of a counter jet may arise in the process of bubble collapse. There has not been a firm conclusion for the exact characteristics which cause the destruction of the surface on the solid boundary.

Rayleigh (1917) studied the corrosion of high speed blades subjected to the effect of a cavitation bubble. He mentioned that the bubble collapse is able to produce a high speed jet flow that damages the solid surface. During 
the course of his research, he developed the pressure dynamic theory for the collapse of spherical bubbles and derived the Rayleigh equation. Many researchers since carried out related researches based on this theory. Among those researchers are Plesset (1949), who further considered the influence of the physical characteristics of fluid viscosity and surface tension and derived the RayleighPlesset equation. Gilmore (1952) moved forward to consider the influence of the compressibility of fluid on the flow field of the bubble collapse. Plesset and Zwick (1952) furthered the research to include the influence of the thermo conductivity of the fluid flow field of the bubble collapse. According to their research results, the time required for the bubble collapse is too short, and the influence of the thermo conductivity is kept at minimum. Therefore, the bubble collapse can be assumed to be a heat adiabatic process.

Kornfeld and Suvorov (1944) brought up the theory of bubble collapse near a solid boundary. They proposed that the bubble would be deformed to a non-spherical shape with the involution of its surface which subsequently generates the phenomenon of jet flow. This phenomenon was proved in the experiment carried out by Naude and Ellis (1961). The numerical model in Plesset and Chapman (1971) research also revealed this phenomenon. If the solid boundary is located on the right side of the bubble, the jet flow would be formed on the left side of the bubble and deform it before arriving at the right side surface of the bubble. The damage of the solid boundary might be caused by the impact of this jet flow during the bubble collapse. Benjamin and Ellis (1966); Philipp and Lauterborn (1998) also detected the bubble collapse phenomenon and its resultant behavior of damage at the solid boundary. Recent research results revealed that the destructive power of the jet flow was not the main factor for the damage of the solid boundary. However, the jet flow influence that causes the collapse of the bubble is still an important element for the research of the hydrodynamics of the flow field.

Rayleigh (1917) first analyzed the theoretical pressure variation of the flow field of the bubble collapse. The bubble collapse results in very high pressure, forming a shock wave which is sent toward the outside of the bubble. Harrison (1952) in his experimental results proved the existence of noise generated by the collapse of a bubble at its surrounding solid boundary. Vogel and Lauterborn (1988) found a close relationship between the strength of the wave pulse and the distance between the bubble and the solid boundary. This wave pulse could then generate a series of shock waves. This phenomenon was studied and revealed in the experiments carried out by Tomita and Shima(1986); Ward and Emmony (1991); Ohl et al. (1995); Shaw et al.(1996); and Lindau and Lauterborn (2003).
Light can be emitted in the process of the bubble collapse when the volume of the bubble is compressed to its minimum size during which the gas inside is heated in an adiabatic process. For bubbles under low viscosity and high pressure, light emission is easier. This is because at high viscosity, the time for bubble collapse is increased and the gas inside is not heated to a sufficient temperature to emit light. On the other hand, for bubbles under lower fluid viscosity, their volume could be extended easily for the emission of light. Ohl et al. (1998) also found the emission of light near the solid boundary under specific conditions in the process of bubble collapse. This phenomenon is called "Single Cavitation Bubble Luminescence" (SCBL). Buzukov and Teslenko (1971); Akmanov et al. (1974) also had similar research reports. The strength of the SCBL is closely related to the distance between the bubble and its surrounding solid boundary (Ohl et al. 1999). This relationship might be a result of the compressibility (under the influence of the distance to the solid boundary) of the bubble. The researches related to the SCBL in recent years include Wolfrum et al. (2001) and Baghdassarian et al. (2001).

A counter jet can be generated when the bubble is located near a solid boundary. The initial formation and increment of the size of the counter jet is very rapid and it can exist for a while. Experiments related to the counter jet are found in Harrison's (1952) and Kling and Hammitt's (1972) researches, but the counter jet phenomenon was not described until Lauterborn (1974). There has not been a final conclusion for the cause of the generation of the counter jet. A counter jet did not appear in the numerical simulations carried out by Best (1993); Zhang et al. (1993); and Blake et al. (1997). However, it appeared in the experiments carried out by Tomita and Shima (1986); Vogel et al. (1989); Ward and Emmony (1991); Philipp and Lauterborn (1998); and Kodama and Tomita (2000). The discrepancy between the numerical simulations and the experimental results leads to the assumption that the counter jet flow field is not part of the bubble collapse process. Its formation might be due to a complicated mechanism in the fluid during the bubble collapse. For example, if the bubble is in contact with the solid boundary, the counter jet would not be generated. The shock wave generated appears at the final stage of the process of bubble collapse. Because the counter jets also appear at the final stage of the bubble collapse, there are speculations that they form due to shock wave structure.

In the experiments carried out by Vogel et al. (1989), the appearance of the counter jet during the bubble collapse is dependent on the distance from the center of the bubble to the solid boundary: 
$\gamma=\frac{d}{R_{\max }}$,

where $R_{\max }$ is the maximum radius of the bubble and $d$ is the distance between the bubble center and the solid boundary. When $\gamma$ is in the range of $1<\gamma \approx 3$, a counter jet could be observed. However, no counter jet is generated under the condition of $\gamma>3$. Lindau and Lauterborn (2003) discussed the relationships between the rebound height, the collapse time and their respective $\gamma$ values in the phenomenon of counter jet. These results revealed an increasing $\gamma$ for a smaller rebound height, and a shorter time of collapse.

Best (1993) introduced a numerical simulation method for the process of bubble collapse. Tong et al. (1999) presented a simulation of the flow field of bubble collapse at different positions. In their analysis, it appeared that no counter jet was formed when the bubble collapsed in the range of $0.6<\gamma<1$. Instead, a splash would be produced after the liquid jet deformed the bubble surface and subsequently hit the solid boundary. The pressure generated by the splash could have considerable influence on the boundary. The first experimental evidence of such an effect was based on the pressure measurements by Shaw et al. (2001). Brujan et al. (2002) used a high speed camera to present a series of images of the toroidal bubble collapse with the splash located in the range between $\gamma=1.1$ and 0.9 . This result was compared with the numerical simulations. Lindau and Lauterborn (2003) also presented a series of experimental results regarding the splash in order to obtain an agreement with the numerical simulations. When $\gamma$ was $<0.6$, the fluid layer between the bubble surface and the solid boundary was too thin to form the splash.

According to Rayleigh's equation, when the effect of the surrounding solid boundary is excluded, the relationship between the time of bubble collapse and its radius is:

$R_{\max }=1.09 \sqrt{\frac{p-p_{v}}{\rho}} t_{c}$,

where $p$ and $\rho$ are the pressure of the flow field and the fluid density at ambient temperature, respectively, $p_{v}$ is the vapor pressure, $t_{c}$ is the bubble collapse time. If the solid boundary condition is put into consideration, a greater bubble collapse time is required. Generally, the size of a cavitation bubble produced in the laboratory is about $1.5 \mathrm{~mm}$ in radius. Under ambient temperature, the bubble collapse time ranges from 100 to $200 \mu$ s. It is not easy to generate cavitation bubbles for their small volumes, short collapse times, and complicated flow fields; all of which contribute to a great difficulty of the measurement. In order to record and analyze the characteristics of the flow field of the bubble collapse, common experimental setup includes a high speed camera with framing rates ranging between several thousand to 100 million frames per second. Some researchers used the method of particle image velocimetry (PIV) to measure the velocity flow field of the process of bubble collapse (Vogel and Lauterborn 1988). However, since the volume of the bubble was small and its collapse time was too short, only a rough sketch of the flow field was obtained. Lawson et al. (1999) applied the PIV method to measure the flow field of the collapse of an $80 \mathrm{~mm}$ diameter rubber balloon and compared it with the numerical simulation. Although these results obtained agreement, there is a great discrepancy between the flow field features of the collapse of a balloon and a bubble. Jaw et al. (2007) obtained sound experimental results using soap bubbles filled with smog particles and applied the PIV method to measure flow fields at different phases during the process of bubble collapse.

In laboratory experiments, Lauterborn (1969) utilized a rotating centrifuge device to generate cavitation bubbles by tearing degassed and partially degassed distilled water columns with calculated tensile strength. For the partially degassed distilled water column, the cavitation bubble was generated at the central axis of rotation by tearing the water column using an accelerated centrifuge. The size of the bubble decreased when the rotation of the centrifuge was decelerated. A cavitation bubble remained in the water column when the centrifuge came to a complete stop. This bubble would wither and completely dissolve into the water column within a few days. For the degassed distilled water column, the cavitation bubble was also generated at the central axis of rotation by tearing the water column using an accelerated centrifuge. Initially, a small bubble appeared at the central axis of rotation near the glass surface. Since the angular velocity increased, other small bubbles appeared near the central axis of rotation and merged to form one large cavitation bubble when the water column was completely torn apart by the tensile strength induced by the rotation. The bubble decreased in size and collapsed when the rotation of the centrifuge was decelerated to a complete stop. It was discovered that higher tensile strength was required to tear a degassed distilled water column compared with a partially degassed one. In addition, when tearing the same degassed distilled water column, the tensile strength required was found to increase with the number of experiments repeated. Minor fissures, cracks, and nuclei on the glass surface that trap tiny bubbles were found related to this increase in tensile strength. When these nuclei and tiny bubbles were released into the water column during the rotation, the fissures and cracks on the glass surface were filled by water, thus, requiring a higher tensile strength to tear the water column.

On the other hand, a single cavitation bubble can be generated in a cuvette using a high energy laser beam to 
focus on a single point (Lauterborn 1972, 1974). In the following years, many related studies utilized this method to generate a single cavitation bubble. Since these bubbles were generated by the high energy laser beam which causes fluid decomposition, it was restricted by the strength of the energy provided by the laser. Usually, the bubble created using this method has a small volume with $1.5 \mathrm{~mm}$ in radius. In addition, the inside pressure of the bubble was not equivalent to the vapor pressure at ambient temperature. Some other researchers used the method of electrolysis to generate a bubble on a platinum electrode at the bottom of a cuvette. However, this method has the defect of disturbing the flow field during the bubble collapse. Another method for forming the bubble is through the use of a needle to inject air into a cuvette before using a lithotripter to generate a shock wave up to $94 \mathrm{MPa}$ to compress the bubble (Philipp et al, 1993). Sankin et al. (2005) also used a lithotripter to generate a $39 \mathrm{MPa}$ shock wave to compress the laserinduced bubble in order to measure the flow field of the interaction between the bubble collapse and the shock wave.

From the researches mentioned above, it can be perceived that the flow field of a cavitation bubble collapse is very complicated in addition to the difficulties of its generation. The cavitation bubble has a small volume and collapses in a relatively short period of time. Even when an expensive high speed camera device is used with framing rates more than 100 million frames per second, there are still many limitations for the measurement of the flow field characteristics of the bubble collapse. Many causes for this complicated phenomenon are still unclear. Moreover, utilization of a high energy laser beam or a platinum electrode method for the generation of cavitation bubbles are also different from the bubbles generated by lowering the fluid pressure to approximately the vapor pressure. A real cavitation bubble containing vapor would produce a greater energy during its collapse than the ones with non-condensable gas (Akmanov et al. 1974; Zhu and Zhong 1999). In this study, a rotational U-shape platform device is utilized to generate a cavitation bubble by the centrifugal force at the center of the rotational axis. Pressure wave of different strengths are used to impinge the bubble at different distances between the bubble and the solid boundary while the flow field features are analyzed. In addition, the use of a low strength pressure wave for the compression of the cavitation bubble could extend the bubble collapse time, and, therefore, lead to a clearer presentation of the flow field features in the process of bubble collapse. This study emphasizes the discussion and description of the causes of the phenomenon of deformation of cavitation bubbles, and the generation of the liquid jet and the counter jet.

\section{Experimental arrangement and cavitation bubble generator}

The experimental setup for the flow field measurement of cavitation bubble collapse is shown in Fig. 1. This device consists of an insulated optical platform, a motor, a rotatable U-shape platform, a transparent cylindrical tube, a set of light sources, a shock wave pressure generator, a high speed camera, and a pressure sensor. The LEEDAN DC brushless motor is capable to produce a maximum controlled angular velocity up to 2,000 RPM.

The U-shape platform was made up of an acrylic platform of $20 \mathrm{~mm}$ in thickness. Centered at the rotational axis of the motor, its rotatable arm has a radius of $250 \mathrm{~mm}$, which results in a total horizontal length of $500 \mathrm{~mm}$. Two vertical forearms each of $150 \mathrm{~mm}$ in height are fixed to the edge of the platform. On the platform of the horizontal

Fig. 1 Schematic diagram of the experiment setup

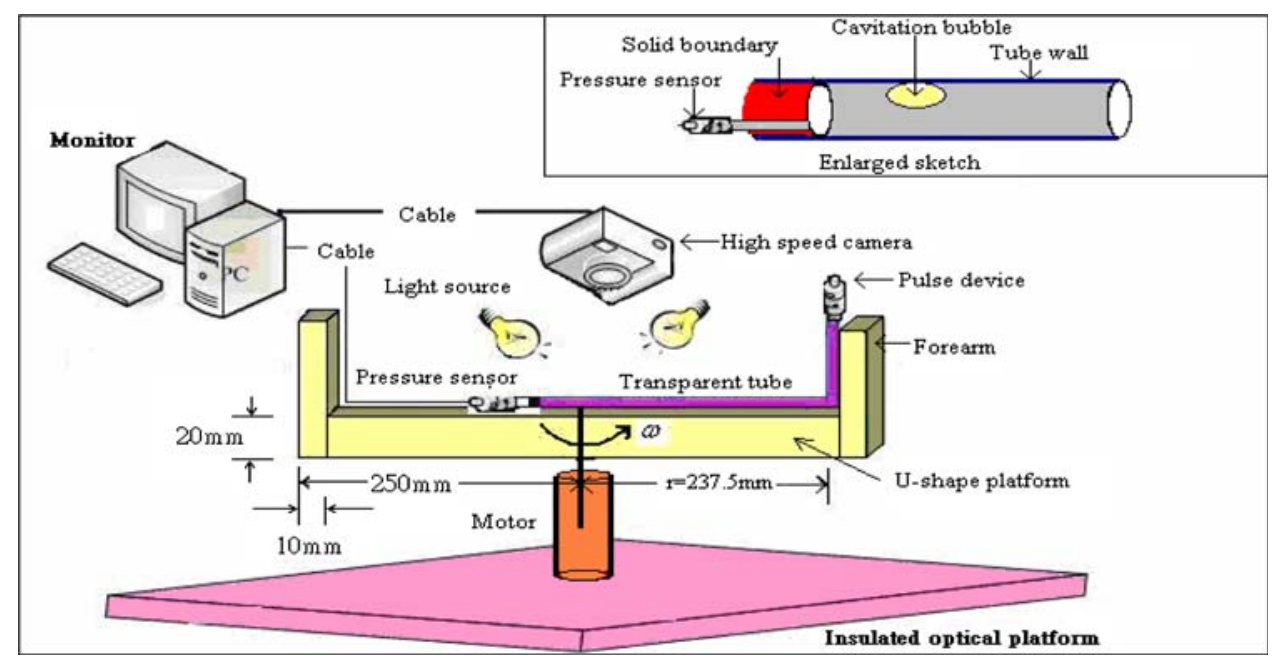




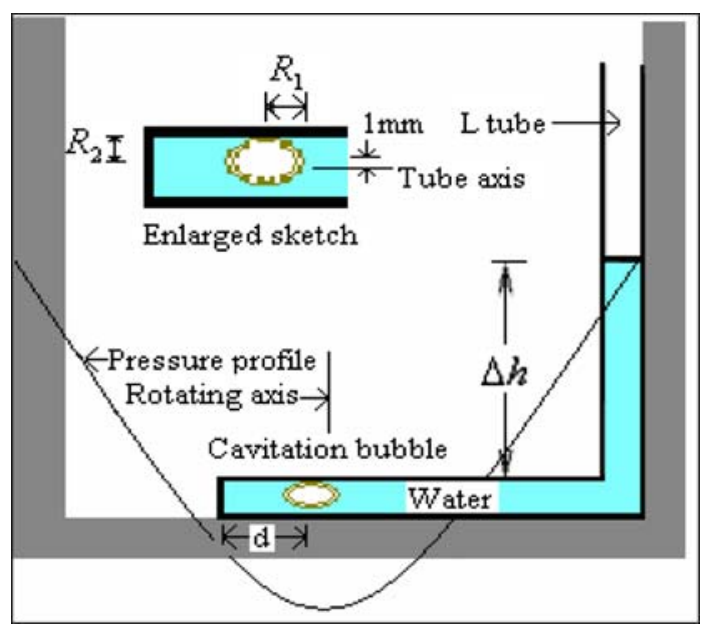

Fig. 2 The pressure distribution for a rotating U-shape platform, where $R_{1}=R_{\max }$ and $R_{2}$ : the height between the bubble center and upper tube wall $\left(R_{1} \leq d \leq 7 R_{1}\right)$

rotatable level arm sits the transparent cylindrical tube of $200 \mathrm{~mm}$ in length, with its internal and external diameter of 5 and $8 \mathrm{~mm}$, respectively. A soft PVC tube with an internal diameter of $5 \mathrm{~mm}$ is fixed to the vertical forearm in order to conveniently exchange the experimental equipment. The center of the soft PVC tube distance to the rotational axis is $237.5 \mathrm{~mm}$. At one end, this tube is connected to the shock wave pressure generator with a piston while it is extended to connect the transparent cylindrical tube at the other end. At the extremity of the transparent cylindrical tube, a $1 \mathrm{~mm}$ diameter hole is drilled on the corner of the solid boundary where its influence to the induced bubble collapse flow can be kept at minimum (shown at the upper part of Fig. 1). On the other hand, the cavitation bubble generation takes place at the location on the platform of the rotational axis where the pressure is at the lowest. Therefore, the transparent cylindrical tube must be located across the center of the rotational axis for easier cavitation bubble generation.

During the experiment of generating a single cavitation bubble, the transparent cylindrical tube on the U-shape platform is filled with tap water shown in Fig. 2. Tap water was used so that true cavitation bubble can be generated. The surface of the fluid at the part of the vertical forearm tube is in touch with air with one atmospheric pressure. Therefore, the center location of the L-tube at initial condition has a hydrostatic pressure of $p_{0}=p_{\text {atm }}+\rho g \Delta h$, where $p_{\text {atm }}$ is the atmosphere pressure, $g$ is the acceleration of gravity, and $\Delta h$ is the water depth difference.

When the U-shape platform is rotated by the motor, the fluid is subjected to a centrifugal force resulting in a parabolic fluid pressure distribution shown as the solid line in Fig. 2 at a different radius. At the vertical forearm, although the height $\Delta h$ is slightly increased, the hydrostatic water pressure is still kept at one atmospheric pressure because the water surface is still in touch with the air. Therefore, the pressure difference between the free surface atmospheric pressure and the pressure at the center of rotation is $\rho g \Delta h-\frac{1}{2} \rho r^{2} \omega^{2}$, where $r$ is the rotational radius and $\omega$ is the angular velocity.

When $\omega$ is gradually increased, the pressure at the center of the rotation in the transparent cylindrical tube is gradually decreased to a saturated vapor pressure at local water temperature. At this condition, a single cavitation bubble near the rotating axis can be generated. A rotating U-tube was used in Lauterborn's (1969) experiment to generate a cavitation bubble which confirmed that the bubble generated is a cavitation bubble. The angular velocity needed for generating a cavitation bubble is related to the height $\Delta h$. Greater $\Delta h$ means a greater angular velocity required for the production of a cavitation bubble. If $\Delta h$ is kept constant, an increasing angular velocity would result in a greater cavitation bubble size. Therefore, by controlling the angular velocity of the U-shape platform, a desirable size of a single cavitation bubble could be generated.

After the cavitation bubble is generated, the U-shape platform is stopped to restore the pressure back to the hydrostatic pressure. The time required to fully stop the rotating U-shape platform is estimated within $2 \mathrm{~s}$. The cavitation bubble generation located nearly rotational axis and the height between the center of the cavitation bubble and tube axis is nearly $1 \mathrm{~mm}$ as shown in Fig. 2 indicated by the enlarged sketch. This pressure difference alone is not enough to collapse the cavitation bubble. To observe the flow field of the collapse of the cavitation bubble, this study uses a pulse setup to hit the piston of the PVC soft tube in contact with the free water surface and instantly generates a shock wave pressure that sends a pulse to cause the collapse of the cavitation bubble.

A Fastec high speed camera is used to extract and record the experimental images. The speed of image extraction is determined by the size of the image. For example, an image extraction speed of 4,000 frames/s is used for an image size of $1,280 \times 128$ pixels. A Kulite XTL-190 pressure sensor together with the NATIONAL INSTRUMENTS (NI)-6221 Analog I/O device is used for the measurement of the pressure profile. The NI-6221 Analog $\mathrm{I} / \mathrm{O}$ device can send a $10 \mathrm{~V}$ signal to drive the pressure sensor and receive a $0-0.5 \mathrm{~V}$ pressure signal to record data for the analysis of the pressure change profile in the transparent cylindrical tube.

In addition, the image data are related to the pressure change profile on the basis of the real-time data acquisition. For each image taken by the high speed camera, a $3.2 \mathrm{~V}$ signal is sent simultaneously from the external output of the camera through a coaxial cable to the NI-6221 device at the receiving end. Through this $\mathrm{I} / \mathrm{O}$ function, a signal is sent to 
trigger the pressure sensor and finally a pressure signal is sent to the I/O function for recording. When the high speed camera stopped recording the image files, the pressure sensor simultaneously stopped extracting data. Hence, every recorded image of the cavitation bubble in its collapse process can be matched with the measured pressure data from the pressure sensor for the recognition of image data with the pressure change profile in the transparent cylindrical tube before taking these data for further analysis.

\section{Flow field measurement of the collapse of cavitation bubbles}

To investigate the characteristics of the liquid jet and the counter jet formed in the bubble collapse flow, a series of experiments with different $\gamma$ 's are performed respectively. Pressure waves of different strengths are applied to induce the bubble collapse flow. The experimental results are discussed below

\subsection{Liquid jet formation}

To manifest the formation of the liquid jet at the central axis of the cavitation bubble, experiments were carried out to generate a $3.5 \mathrm{~mm}$ radius cavitation bubble on a $30 \times$ $30 \times 1.2 \mathrm{~mm}$ (length $\times$ width $\times$ depth) platform located at the center of the rotational axis to approximately simulate a two-dimensional cross-sectional view of the bubble collapse process with a pressure wave of $60 \mathrm{kPa}$. During the formation of the inward dent, a liquid jet was formed at the central axis of the cavitation bubble as shown in Fig. 3.

Initially, the liquid jet is converged as the bubble surface concaved toward the center of the bubble. The left hand side bubble surface progressively moves toward the right hand side surface of the bubble. The counter force opposing the liquid jet is then gradually increased as the two bubble surfaces approach each other. At the same time, the liquid jet is accumulating energy and forming a structure that has a larger front and a smaller rear, as shown in the second row of Fig. 3. From these series of images, the features of the liquid jet formation without solid boundary effect are clearly manifested.
3.2 Flow field measurement of bubble collapse at $\gamma \approx 7$

Under this condition, the distance between the center of the cavitation bubble and the solid boundary is nearly seven time of its $R_{\max }$. The flow field of the process of cavitation bubble collapse is not affected by the solid boundary. Therefore, the solid boundary is assumed to be insignificant to the process of bubble collapse. This process of the cavitation bubble being pressurized followed by its final collapse is shown in Fig. 4. The pressure wave is sent from the left side of the bubble surface, impacting the bubble with peak strength of $155 \mathrm{kPa}$. The pressure wave caused an inward dent deformation of the bubble shown in the first row of Fig. 4. Figure 5 shows the variation of pressure with time. The pulse setup was employed to hit the piston that moved $2.5 \mathrm{~mm}$ in distance to instantaneously form a pressure wave which compressed and collapsed the bubble. The piston used to generate the pressure wave is about the same size of the tube. Because the generated pressure covers the whole area of the tube, the pressure measured is equivalent to the average pressure inside the tube. The width of the pressure wave generated is larger than the bubble size so that the indent covers the whole diameter of the bubble. The pulse durations are $2.75 \mathrm{~ms}$ for both the compressive and tensile pressure waves.

When sufficient energy is accumulated by the liquid jet during its continuous motion to the right side of the bubble, the overlaid bubble surface is squeezed and subsequently spouted into a jet flow. When the jet flow extended to the static fluid at the right side of the bubble, rapid variation in the flow velocity is created which leads to a KelvinHelmholtz vortex shown in the second and third rows of Fig. 4. Jaw et al. (2007) clearly demonstrated the KelvinHelmholtz vortex formation in their measurements of soap bubble collapse flow. The bubble penetrated by the jet flow was then torn into two bubbles by the Kelvin-Helmholtz vortex. If the strength of the pressure is increased, the bubble could be separated into a number of smaller bubbles. From these series of images of the cavitation bubble collapse, it can be perceived that pressure wave initiates bubble collapse with liquid jet formation.

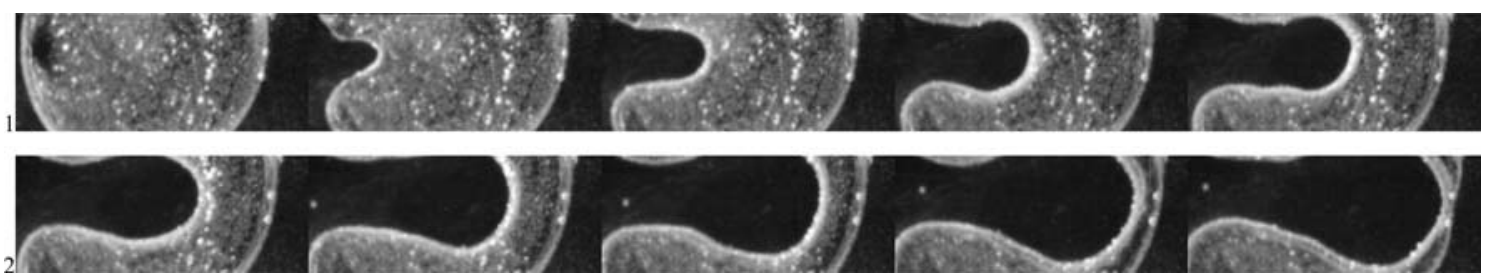

Fig. 3 Liquid jet accumulating energy in the inward dent formed a larger front and a smaller rear shape. The peak strength of the pressure wave is $60 \mathrm{kPa}$. Image interval time is $1 / 100 \mathrm{~s}$. The size of each individual frame is $8.0 \times 3.0 \mathrm{~mm}$. The bubble $R_{\max }$ is $3.5 \mathrm{~mm}$ 


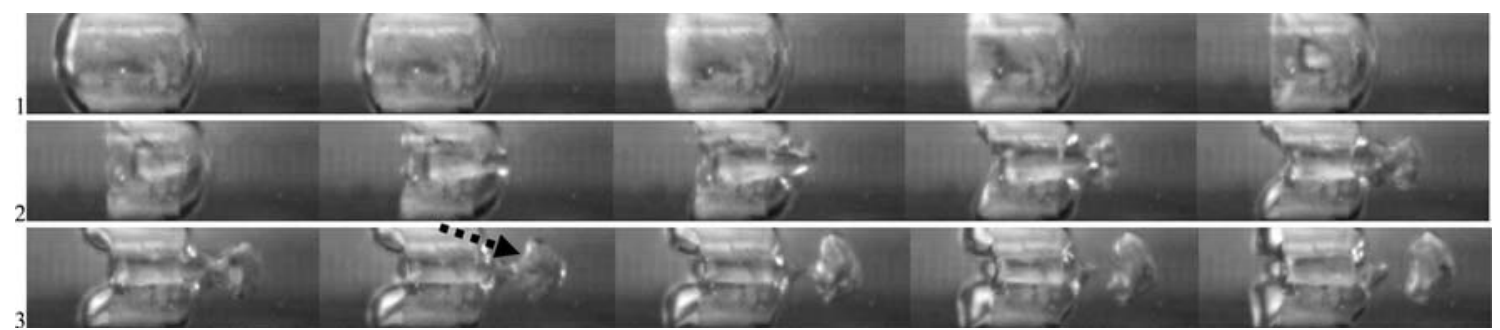

Fig. 4 Top view of images of the process of bubble collapse at $\gamma \approx 7$. First row: image of the inward dent process; second and third rows: images of the Kelvin-Helmholtz vortex process (the KelvinHelmholtz vortex is indicated by a dotted line with an arrow). The peak strength of the pressure wave is $1,55 \mathrm{kPa}$. Image interval time is
$1 / 2,000 \mathrm{~s}$. The size of each individual frame is $11.5 \times 3.1 \mathrm{~mm}$. The bubble $R_{\max }$ is $2.5 \mathrm{~mm}$. The diameter of initial jet flow size $\left(D_{j}\right)$ is $1.1 \mathrm{~mm}$ in the third image at the second row. The outer diameter of the vortex ring $\left(D_{v \max }\right)$ is $2.1 \mathrm{~mm}$ in the third image at the third row. $(\Delta h: 55 \mathrm{~mm}, \omega: 200 \mathrm{RPM})$
Fig. 5 The pressure wave is comprised of a compressive wave with a peak pressure of $155 \mathrm{kPa}$ followed by a tensile wave of $-25 \mathrm{kPa}$ in peak pressure. The pulse durations are $2.75 \mathrm{~ms}$ for both the compressive and tensile pressure waves.

$\left(\Delta p=p_{\mathrm{m}}-p_{\mathrm{atm}}, p_{\mathrm{m}}:\right.$ measured value)

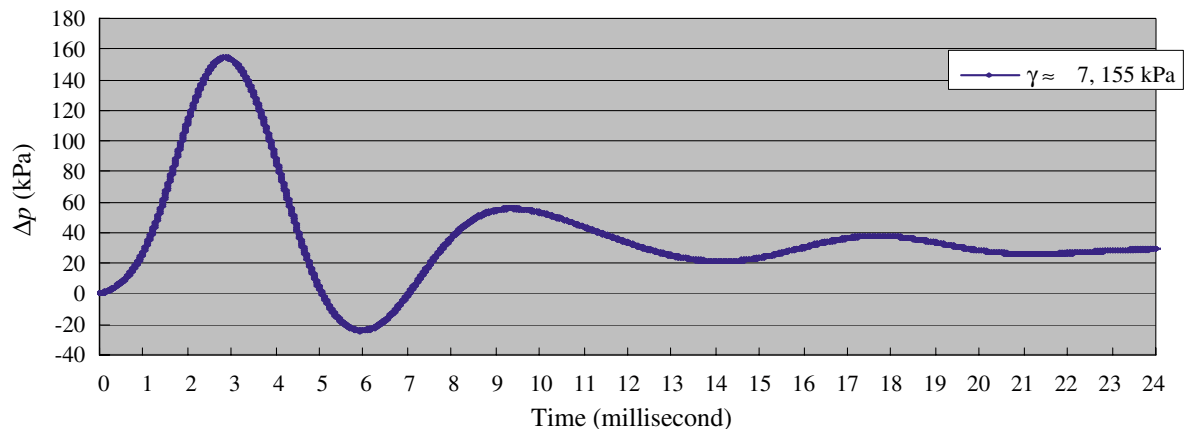

The surrounding boundary effects are difficult to analyze without quantitative measurements, such as using PIV method However, for the experiments conducted in this study, the width of the pressure wave generated is larger than the bubble size, and pressure is uniformly distributed across the tube area. The bubble collapse is caused by the pressure difference across the bubble surface, and the induced flow is along the axis of the tube, not normal to the surrounding boundary. Therefore, the tube boundary seems not to introduce significant influence on the axial flow induced by the bubble collapse. The vortex formed in Fig. 4 clearly revealed such phenomena. Sankin et al. (2005) also performed experiments of shock wave interaction with single bubble in a small water tank; no significant effects of the surrounding boundary were reported either.

\subsection{Flow field measurement of bubble collapse at $\gamma \approx 2$}

As described in the introduction, the counter jet will be generated when the distance between the center of the bubble and the solid boundary is within one to three times the bubble's radius $(1<\gamma \approx 3)$. The experiments conducted with $\gamma \approx 2$ falls within this range.

The distance from the right side of the bubble surface to the solid boundary is only one radius long. The KelvinHelmholtz vortex was generated after the liquid jet impinged the bubble surface and the jet flow is formed. This vortex would touch the solid boundary and subsequently form a stagnation ring on the solid boundary shown in the top view sketch in Fig. 6. After the stagnation ring touched the solid boundary, it was divided into two fluid flows. One of them was outside the stagnation ring splashing outwardly along the radial direction. The other fluid flow inside the stagnation ring was squeezed inwardly to form a counter jet shown in the side view sketch in Fig. 6. For a liquid layer to exist between the bubble surface and the solid boundary, the distance from the bubble center to the solid boundary must be larger than the bubble radius, or $\gamma$ must be larger than one. Therefore, $\gamma>1$ is a necessary condition for the bubble collapse flow to generate the counter jet.

On the other hand, after the bubble surface was penetrated to form the Kelvin-Helmholtz vortex, a zone with high velocity and low pressure was formed at the root of the central axis of the vortex where the bubble was stretched and deformed towards its right side shown in the images at the first and second rows of Fig. 6. From the third row to the fourth row images of Fig. 6, a fully developed counter jet process located at the central axis of the bubble could be clearly seen.

Many researchers who studied the counter jet have mentioned the existence of the stagnation ring. However, in these studies, the time for the collapse of the bubble was too short for the appearance of the Kelvin-Helmholtz 

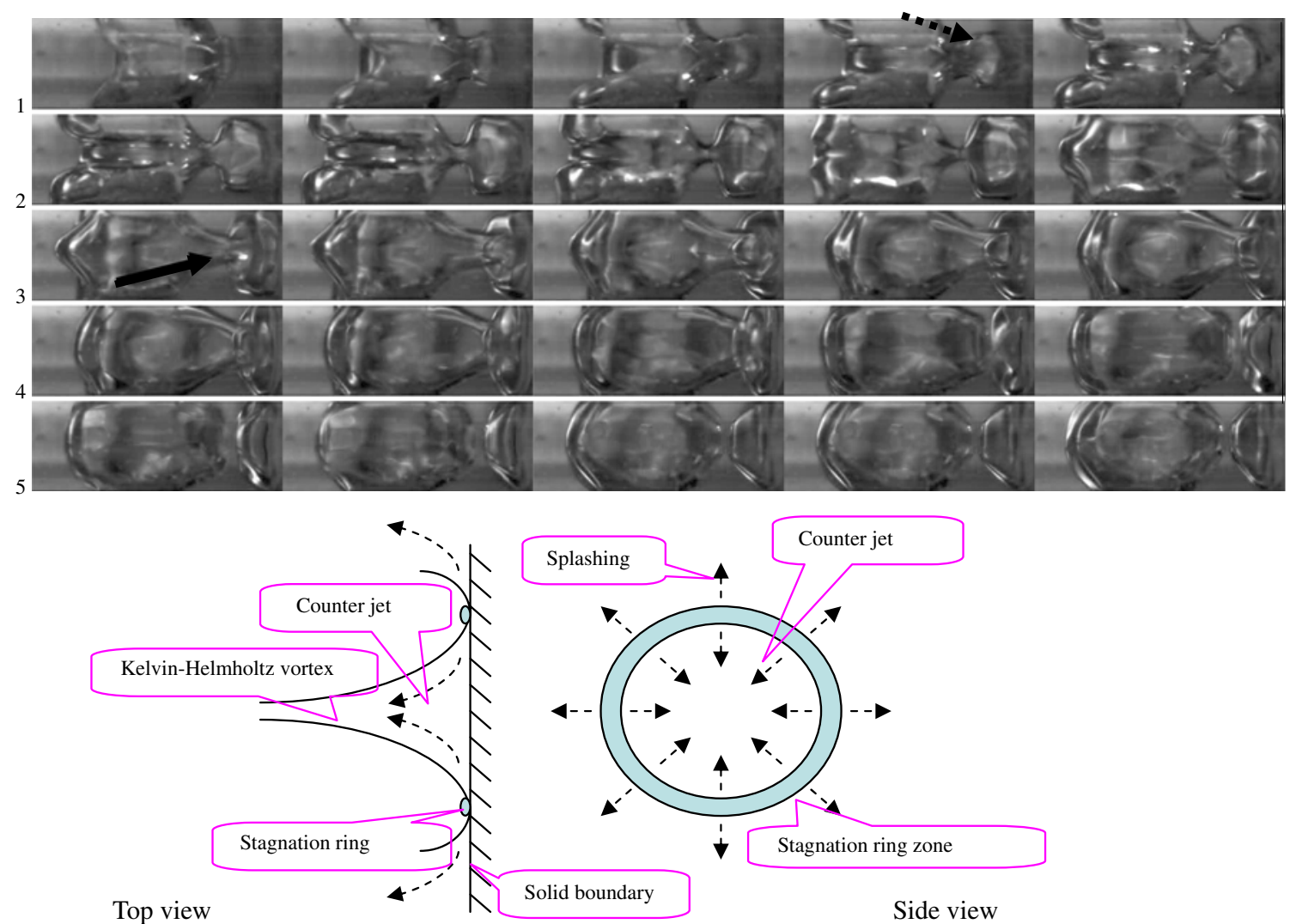

Fig. 6 Upper part the process of bubble collapse at $\gamma \approx 2$ (the Kelvin-Helmholtz vortex is indicated by a dotted line with an arrow, the counter jet indicated by a solid line with an arrow). The peak strength of the pressure wave is $260 \mathrm{kPa}$. The image time interval is $1 / 2,000 \mathrm{~s}$. The size of each individual frame is $8.5 \times 3.1 \mathrm{~mm} . R_{\max }$ is

vortex. The relationship between the stagnation ring and the Kelvin-Helmholtz vortex was still not clear. In this study, the process for the formation of the Kelvin-Helmholtz vortex and the counter jet was clearly revealed for a lower pressure wave utilized to compress the cavitation bubble. If the strength of the pressure wave is increased, the resultant counter jet could penetrate the cavitation bubble and subsequently separate the bubble into a number of bubbles as shown in Fig. 7. In the fourth image at the
$2.5 \mathrm{~mm} . D_{j}$ is $1.1 \mathrm{~mm}$ in the first image at the first row. $D_{v \max }$ is $2.6 \mathrm{~mm}$ in the first image at the second row. $(\Delta h: 55 \mathrm{~mm}, \omega: 200$ RPM) Lower part: sketch of Kelvin-Helmholtz vortex forming the counter jet (note: The light area at the bubble surface is the reflection light shown from the third image to fifth image at the second row)

second row of Fig. 8, a fully developed counter jet located at the central axis of the bubble can be seen.

\subsection{Flow field measurement of bubble collapse} at $\gamma \approx 3$

The $\gamma \approx 3$ is a critical value for the generation of a counter jet. In this study, three different strengths of pressure waves are used to induce the cavitation bubble collapse.

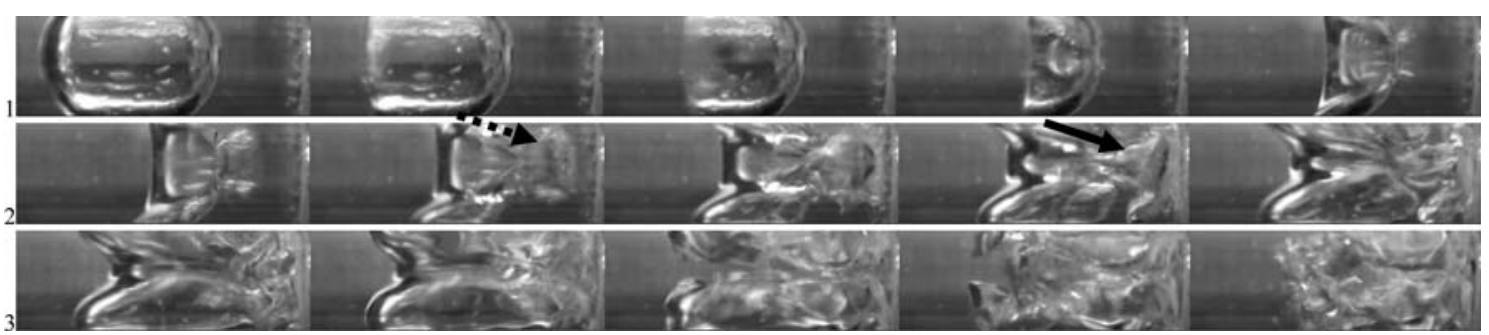

Fig. 7 Images of the process of bubble collapse at $\gamma \approx 2$ with image time interval of $1 / 2,000 \mathrm{~s}$. The peak strength of the pressure wave is $405 \mathrm{kPa}$. The size of each individual frame is $9.8 \times 3.1 \mathrm{~mm}$ (the Kelvin-Helmholtz vortex is indicated by a dotted line with an arrow; the counter jet indicated by a solid line with an arrow). $R_{\max }$ is $2.5 \mathrm{~mm} . D_{j}$ is $1.0 \mathrm{~mm}$ in the fifth image at the first row. $D_{v \max }$ is $2.35 \mathrm{~mm}$ in the second image at the second row. $(\Delta h: 60 \mathrm{~mm}, \omega: 225$ RPM) 

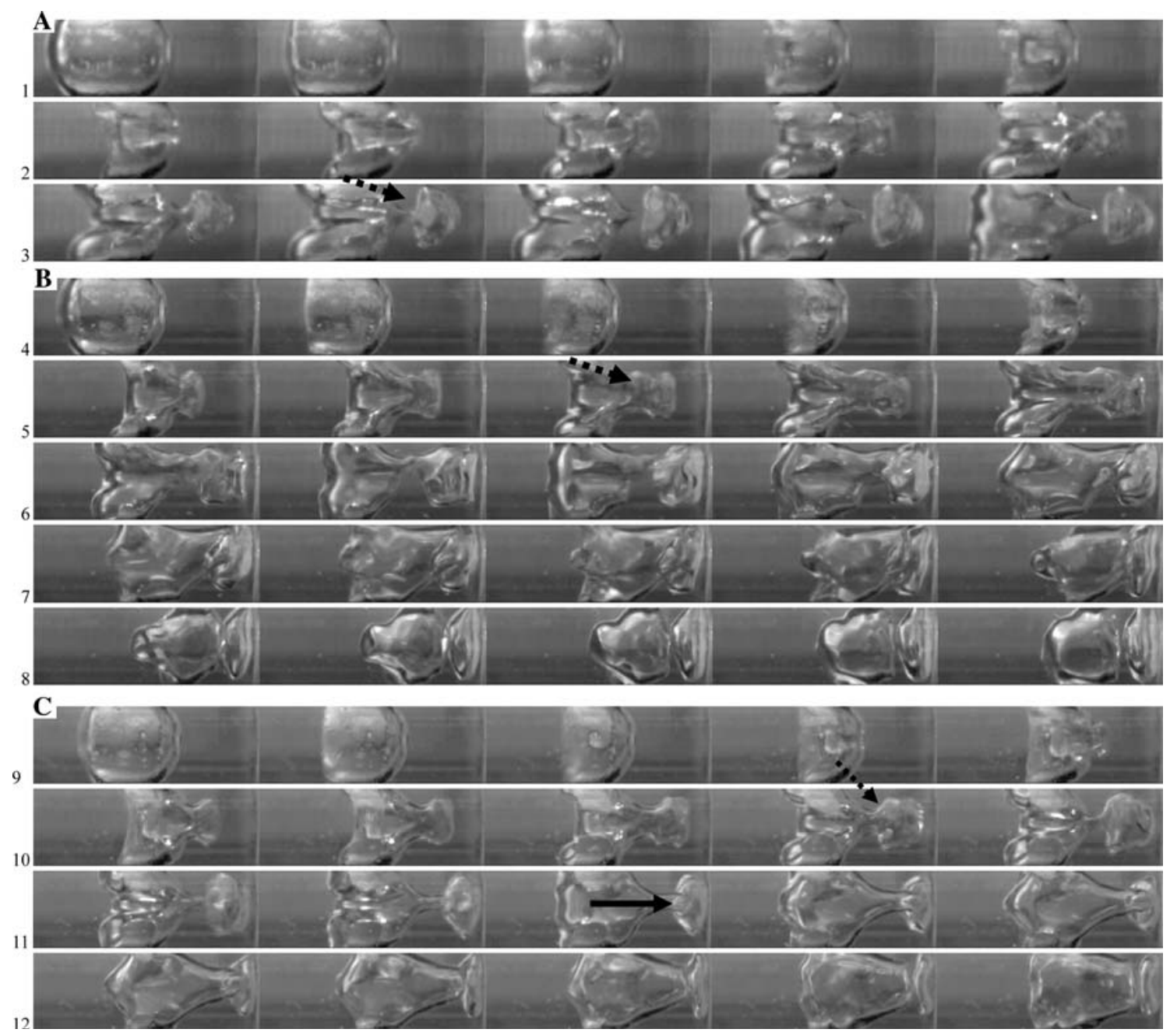

Fig. 8 Images of the process of bubble collapse at $\gamma \approx 3$. The peak strength of the pressure wave for a $200 \mathrm{kPa}$, b $300 \mathrm{kPa}$, c $360 \mathrm{kPa}$. The size of each individual frame for a $10.5 \times 3.1 \mathrm{~mm}$; b $9.6 \times 3.1 \mathrm{~mm}$; c $9.6 \times 3.1 \mathrm{~mm}$. $R_{\max }$ for a 2.45 , b 2.35 , c $2.25 \mathrm{~mm}$. $D_{j}$ for a $1.0 \mathrm{~mm}$ (second image at the second row); b $0.9 \mathrm{~mm}$ (fifth image at the fourth row); c $0.95 \mathrm{~mm}$ (fifth image at the ninth row). $D_{v \max }$ for A: $2.5 \mathrm{~mm}$ (second image at the third row); b $2.0 \mathrm{~mm}$ (third image at the fifth row); c $2.4 \mathrm{~mm}$ (first image at the

The influences of the pressure waves to the formation of the counter jet at this critical stand-off distance are investigated.

The images located from the first to the third rows of Fig. 8 reveal the flow field of bubble collapse under a pressure wave of $200 \mathrm{kPa}$ in strength. An inward dent was formed followed by the generation of a liquid jet which then penetrated the bubble surface to produce the KelvinHelmholtz vortex. The bubble was torn into two bubbles because the Kelvin-Helmholtz vortex did not touch the solid boundary. This process of collapse was similar to the case at $\gamma \approx 7$ where the counter jet was not generated.

The process of the collapse of the bubble, with the strength of the pressure wave being increased to $300 \mathrm{kPa}$, eleventh row). $\Delta h$ for a 60 , b 53 , c $50 \mathrm{~mm}$. $\omega$ for a 225 , b 190 , c $175 \mathrm{RPM}$. The image time interval is $1 / 2,000 \mathrm{~s}$. (The KelvinHelmholtz vortex is indicated by a dotted line with an arrow; the counter jet is indicated by a solid line with an arrow). (Note: a mushroom shape at the central axis of bubble surface is also the Kelvin-Helmholtz vortex shown from the first image to the third image at the tenth row)

is shown from the fourth to the eighth rows in Fig. 8. Unlike the semi-hemispheric form of the Kelvin-Helmholtz vortex shown in the second and third rows of Fig. 8, the vortex shown here was clearly influenced by the solid boundary when the liquid jet compressed the bubble surface. The right side of the head of the vortex touched the solid boundary and turned into a flat shape before splashing and spreading outwardly. On the other hand, before the head of the vortex touched the solid boundary, the outer ring of the vortex had already touched the tube wall and started spreading outwardly as shown in the images at the fifth row in Fig. 9. This spreading vortex kept moving toward the right side until it touched the solid boundary and generated a subsequent shock wave which rebounded to 

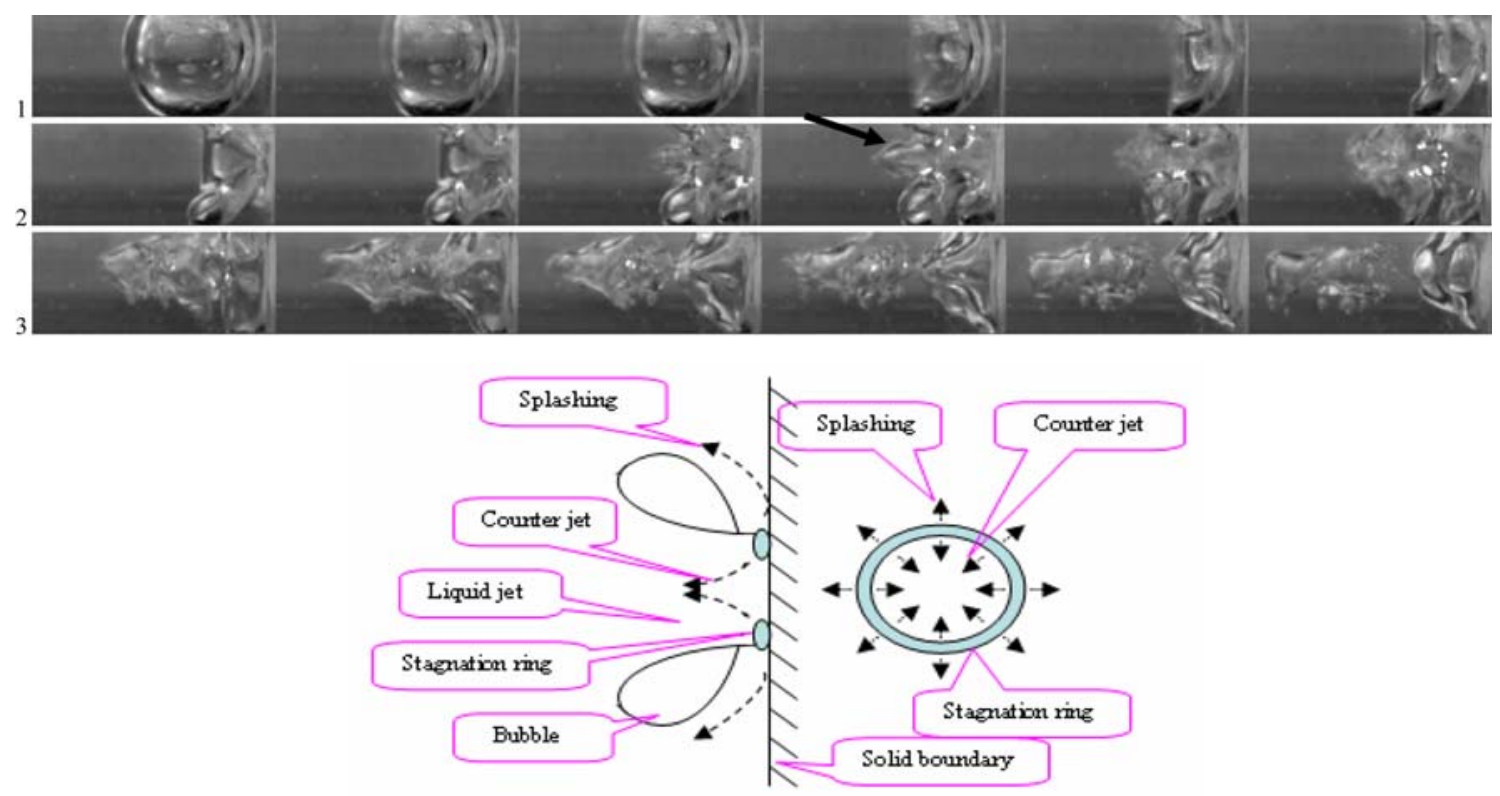

Top view

Side view

Fig. 9 Upper part images of the process of bubble collapse at $\gamma \approx 1$ (the counter jet indicated by an arrow), the peak strength of the pressure wave is $320 \mathrm{kPa}$. The image time interval is $1 / 2,000 \mathrm{~s}$, the size of each individual frame is $8.3 \times 3.1 \mathrm{~mm}$. $R_{\max }$ is $2.4 \mathrm{~mm}$. $(\Delta h$ : $52 \mathrm{~mm}, \omega: 185 \mathrm{RPM}$ ) (Note: a mushroom shape at the central axis of

produce the phenomenon of Richtmyer-Meshkov instability (Meshkov 1969) shown near the solid boundary in every image at the sixth row of Fig. 8. Although the Kelvin-Helmholtz vortex could be generated under this strength of the pressure wave, the vortex had already splashed and touched the surrounding tube wall, disabling the vortex from forming the stagnation ring and the counter jet. At the end of this process, the bubble was torn by the liquid jet and the root of the vortex into two bubbles shown in the images at the seventh and eighth rows in Fig. 9. If the strength of the pressure wave is increased to a peak value of $360 \mathrm{kPa}$, the Kelvin-Helmholtz vortex would touch the solid boundary before the formation of the stagnation ring and the counter jet. This process is shown in the image listed from the 11th to the 12th rows of Fig. 8, and a fully developed counter jet can be seen.

\subsection{Flow field measurement of bubble collapse at $\gamma \approx 1$ and $\gamma=1$}

The other critical value for the formation of the counter jet occurs at $\gamma \approx 1$ where the bubble surface is close to the solid boundary. For this relatively low stand-off distance, a thin fluid layer exists in the small gap between the bubble surface and the solid boundary. In order to understand the characteristics of the flow fields under this critical condition, experiments at locations where $\gamma$ is slightly greater than and equal to one are performed. bubble surface is also the Kelvin-Helmholtz vortex shown from the fourth image to the sixth image at the first row.) Lower part sketch of Kelvin-Helmholtz vortex forming the counter jet (Note: left diagram of lower part: the solid line is the bubble surface and the dotted lines with an arrow are the splashing and the counter jet)

1. The bubble collapse flow induced by a pressure wave of $320 \mathrm{kPa}$ at the stand-off distance $\gamma$ slightly greater than 1 is performed first. The bubble deformed and changed from a bowl-like shape to the toroidal shape after the liquid jet pressurized the bubble surface, as shown in the first row of Fig. 10. Although the gap between the bubble surface and the solid boundary is small, the stagnation ring is still formed after the liquid jet impacts the solid boundary. The outwards radial flow collides with the flow induced by the still contracting bubble and a splash is projected away from the boundary, as shown in the sketch diagram of Fig. 9. The liquid layer in the gap inside the stagnation ring is squeezed inwards to form the counter jet, as shown in the second and third rows and the sketch diagram of Fig. 9. Finally, the bubble is torn into a number of bubbles. Note also that a fully developed counter jet is clearly presented in the third row of Fig. 9.

2. For the stand-off distance $\gamma=1$, the deformed bubble does not become toroidal since the liquid jet cannot thread the bubble surface but just push the front and the rear bubble surfaces to be overlaid on the solid boundary. After the liquid jet impacts the solid boundary, it just splashes along the radial direction. The bubble collapses subsequently along the radial direction without forming the stagnation ring and the counter jet, as the images and the sketch diagram shown in Fig. 10. 

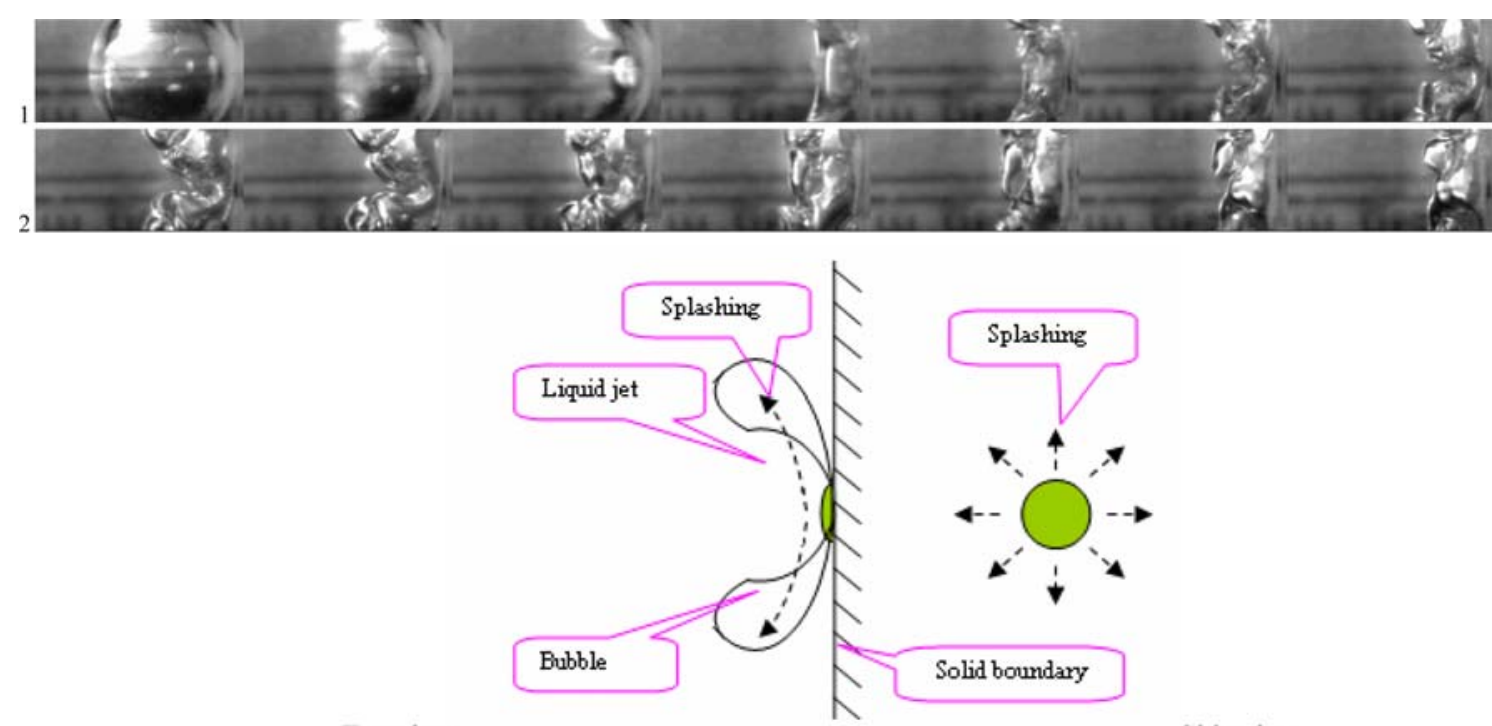

Top view

Fig. 10 Upper part images of the process of bubble collapse at $\gamma=1$; the peak strength of the pressure wave is $520 \mathrm{kPa}$; the image time interval is $1 / 2,000 \mathrm{~s}$. The size of each individual frame is $6.2 \times 3.1 \mathrm{~mm}$. $R_{\max }$ is $2.25 \mathrm{~mm}$. ( $\left.\Delta h: 50 \mathrm{~mm}, \omega: 175 \mathrm{RPM}\right)$ Lower

\section{Conclusions}

This study utilized a U-shape platform device to generate a single cavitation bubble by centrifugal force. The bubble is then collapsed by sending a pressure wave. During the process of the collapse of the cavitation bubble, a high speed camera capable of capturing 4,000 frames/s is used to record the flow field surrounding the collapse of the bubble at different $\gamma$ values. The characteristics of the cavitation bubble collapse flow are clearly manifested by the cinematographic analysis.

For the experiments conducted in this study, the bubble collapse is caused by the pressure difference across the bubble surface, and the induced flow is along the axis of the tube, not normal to the surrounding boundary. The boundary effect of the surrounding is mainly on the rebounded flow after the bubble is fully collapsed, but not during the collapse process when induced jet flow, stagnation ring, and counter jet were forming. Therefore, the uniformly pressure distributed is far more important than the boundary effect for the bubble collapse flow.

In the past studies of the counter jet formational relationship between the stagnation ring and the KelvinHelmholtz vortex was still not clear. In this study, the process for the formation of the Kelvin-Helmholtz vortex and the counter jet was clearly revealed for a lower pressure wave utilized to compress the cavitation bubble.

For a large stand-off distance, $\gamma \approx 7$, the bubble collapsed without solid boundary influence, a liquid jet is formed accompanied with the bubble deformation. The part sketch of the liquid jet position. (Note left diagram of lower part: the solid line is the bubble surface and the dotted line with an arrow is the splashing.)

liquid jet then penetrates the bubble surface. The KelvinHelmholtz instability occurs around the penetrated jet surface and vortices are formed due to the presence of sufficient velocity shear between the jet flow and the surrounding static fluid. Counter jet is not formed for such a stand-off distance.

For the stand-off distance, $\gamma \approx 2$, which falls within the range $1<\gamma \leq 3$, the penetrated jet is capable to impact the solid boundary. A stagnation ring is formed on the solid boundary which separates the jet into outwards and inwards radial flows. The liquid between the bubble surface and the solid boundary is squeezed by the inwards radial flow to form the counter jet.

At the critical stand-off distance, $\gamma \approx 3$, whether the counter jet occurs or not depends on the strength of the pressure wave used to induce the bubble collapse. For a lower strength pressure wave, the liquid jet penetrates the bubble but is not able to impact the solid boundary. Neither stagnation ring nor counter jet can be generated. For an intermediate strength pressure wave, the penetrated jet spreads radially so that the circumference of the jet touches the tube wall before the jet front impacts the solid boundary. Neither stagnation ring nor counter jet can be generated. If the strength of the pressure wave is further increased, the penetrated jet is able to impact the solid boundary to form the stagnation ring and the counter jet.

For the stand-off distance $\gamma$ slightly greater than one, a thin liquid layer exists in the small gap between the bubble surface and the solid boundary. The penetrated jet impacts the boundary directly. The stagnation ring is 
formed on the solid boundary. The thin liquid layer inside the stagnation ring is squeezed by the inwards radial flow to form the counter jet. If $\gamma$ is equal to one, the bubble surface is in contact with the solid boundary, the liquid jet cannot penetrate the bubble but splash along the radial direction without forming the stagnation ring and the counter jet.

For all the experiments performed in this study, the strength of the pressure wave adopted to induce the bubble collapse flow is kept as low as possible so that the bubble collapses in a longer period of time. The characteristics of the bubble collapse flows at different stand-off distances can thus be clearly manifested. However, different strengths of the pressure waves are needed to induce the bubble collapse flow at different $\gamma$ locations. A lower strength of the pressure wave is needed for an increasing $\gamma$ value and vise versa.

Acknowledgments This research is supported by National Science Council, Taiwan under contract No. 96-2221-E-019-056.

Open Access This article is distributed under the terms of the Creative Commons Attribution Noncommercial License which permits any noncommercial use, distribution, and reproduction in any medium, provided the original author(s) and source are credited.

\section{References}

Akmanov AG, Ben'kovskii VG, Golubnichii PI, Maslennikov SI, Shemanin VG (1974) Laser sonoluminescence in a liquid. Soviet Phys Acoustics 19:417-418

Baghdassarian O, Chu HC, Tabbert B, Williams GA (2001) Spectrum of luminescence from laser-created bubbles in water. Phys Rev Lett 86:4934-4937

Benjamin TB, Ellis AT (1966) The collapse of cavitation bubbles and the pressures thereby produced against solid boundaries. Philos Trans Roy Soc Lond Ser A, Math Phys Sci 260:221-240

Best JP (1993) The formation of toroidal bubbles upon the collapse of transient cavities. J Fluid Mech 251:79-107

Blake JR, Hooton MC, Robinson PB, Tong RP (1997) Collapsing cavities, toroidal bubbles and jet impact. Philos Trans Math Phys Eng Sci 355:537-550

Brujan EA, Keen GS, Vogel A, Blake JR (2002) The final stage of the collapse of a cavitation bubble close to a rigid boundary. Phys Fluids 14:85-92

Buzukov AA, Teslenko VS (1971) Sonoluminescence following focusing of laser radiation into liquid. J Exp Theor Phys Lett 14:189-191

Gilmore FR (1952) The growth and collapse of a spherical bubble in a viscous compressible liquid. Technical Report California Institute of Technology, Pasadena, CA

Harrison M (1952) An experimental study of single bubble cavitation noise. J Acoust Soc Am 24:776-782

Jaw SY, Chen CJ, Hwang RR (2007) Flow visualization of bubble collapse flow. J Vis 10:21-24

Kling CL, Hammitt FG (1972) A photographic study of spark-induced cavitation bubble collapse. Transactions of the ASME D. J Basic Eng 94:825-833
Kodama T, Tomita Y (2000) Cavitation bubble behavior and bubbleshock wave interaction near a gelatin surface as a study of in vivo bubble dynamics. Appl Phys B 70:139-149

Kornfeld M, Suvorov L (1944) On the destructive action of cavitation. Journal of Applied Physics 15:495-506

Lauterborn W (1969) Fotografische Aufnahmen vom Aufreißen einer rotierenden Wassersäule und Zerreißfestigkeitsmessungen an Wasser nach der Zentrifugenmethode: Apperatus und erste Ergebnisse. Acustica 22:35-47

Lauterborn W (1972) High-speed photography of laser-induced breakdown in liquids. Appl Phys Lett 21:27-29

Lauterborn W (1974) Kavitation durch Laserlicht. Acustica 31:52-78

Lawson NJ, Rudman A, Guerra J, Liow JL (1999) Experimental and numerical comparisons of the break-up of a large bubble. Exp Fluids 26:524-534

Lindau O, Lauterborn W (2003) Cinematographic observation of the collapse and rebound of a laser-produced cavitation bubble near a wall. J Fluid Mech 479:327-348

Meshkov EE (1969) Instability of the interface of two gases accelerated by a shock wave. Fluid Dyn 4:101-104

Naude CF, Ellis AT (1961) On the mechanism of cavitation damage by nonhemispherical cavities collapse in contact with a solid boundary. Transactions of the ASME D. J Basic Eng 83:648-656

Ohl CD, Philipp A, Lauterborn W (1995) Cavitation bubble collapse studied at 20 million frames per second. Annalen der Physik 4:26-34

Ohl CD, Lindau O, Lauterborn W (1998) Luminescence from spherically and aspherically collapsing laser induced bubbles. Phys Rev Lett 80:393-397

Ohl CD, Kurz T, Geisler R, Lindau O, Lauterborn W (1999) Bubble dynamics, shock waves and sonoluminescence. Philos Trans Roy Soc London Math Phys Eng Sci 357:269-294

Philipp A, Lauterborn W (1998) Cavitation erosion by single laserproduced bubbles. J Fluid Mech 361:75-116

Philipp A, Delius M, Scheffczyk C, Vogel A, Lauterborn W (1993) Interaction of lithotripter-generated shock waves with air bubbles. J Acoust Soc Am 93:2496-2509

Plesset MS (1949) The dynamics of cavitation bubbles. Trans ASME J Appl Mech 16:277-282

Plesset MS, Chapman RB (1971) Collapse of an initially spherical vapour cavity in the neighbourhood of a solid boundary. J Fluid Mech 47:283-290

Plesset MS, Zwick SA (1952) A nonsteady heat diffusion problem with spherical symmetry. J Appl Phys 23:95-98

Rayleigh L (1917) On the pressure developed in a liquid during the collapse of a spherical cavity. Philos Mag 34:94-98

Sankin GN, Simmons WN, Zhu SL, Zhong P (2005) Shock wave interaction with laser-generated single bubbles. Phys Rev Lett 034501:1-4

Shaw SJ, Jin YH, Schiffers WP, Emmony DC (1996) The interaction of a single laser-generated cavity in water with a solid surface. J Acoust Soc Am 99:2811-2824

Shaw SJ, Schiffers WP, Emmony DC (2001) Experimental observations of the stress experienced by a solid surface when a lasercreated bubble oscillates in its vicinity. J Acoust Soc Am 110:1822-1827

Tomita Y, Shima A (1986) Mechanisms of impulsive pressure generation and damage pit formation by bubble collapse. J Fluid Mech 169:535-564

Tong RP, Schiffers WP, Blake SJ (1999) Splashing in the collapse of a laser-generated cavity near a rigid boundary. J Fluid Mech 380:339-361

Vogel A, Lauterborn W (1988) Time-resolved particle image velocimetry used in the investigation of cavitation bubble dynamics. Appl Opt 29:1869-1876 
Vogel A, Lauterborn W, Timm R (1989) Optical and acoustic investigations of the dynamics of laser-produced cavitation bubbles near a solid boundary. J Fluid Mech 206:299-338

Ward B, Emmony DC (1991) Direct observation of the pressure developed in a liquid during cavitation-bubble collapse. Appl Phys Lett 59:2228-2231

Wolfrum B, Kurz T, Lindau O, Lauterborn W (2001) Luminescence of transient bubbles at elevated ambient pressure. Phys Rev E 64:046306-5
Zhang S, Duncan JH, Chahine GL (1993) The final stage of the collapse of a cavitation bubble near a rigid wall. J Fluid Mech 257:147-181

Zhu S, Zhong P (1999) Shock wave-inertial microbubble interaction: a theoretical study based on the Gilmore formulation for bubble dynamics. J Acoust Soc Am 106:3024-3033 